\title{
Exotic exchange bias at epitaxial ferroelectric-ferromagnetic interfaces
}

\author{
Amitesh Paul, ${ }^{1, a)}$ Christoph Reitinger, ${ }^{1}$ Carmine Autieri, ${ }^{2}$ Biplab Sanyal, ${ }^{2}$ \\ Wolfgang Kreuzpaintner, ${ }^{1}$ Jaru Jutimoosik, ${ }^{3}$ Rattikorn Yimnirun, ${ }^{3}$ Francis Bern, ${ }^{4}$ \\ Pablo Esquinazi, ${ }^{4}$ Panagiotis Korelis, ${ }^{5}$ and Peter Böni ${ }^{1}$ \\ ${ }^{1}$ Technische Universität München, Physik Department E21, Lehrstuhl für Neutronenstreuung, \\ James-Franck-Straße 1, D-85748 Garching, Germany \\ ${ }^{2}$ Department of Physics and Astronomy, Uppsala University, Box 516, SE-75120 Uppsala, Sweden \\ ${ }^{3}$ School of Physics, Institute of Science, and NANOTEC-SUT Center of Excellence on Advanced \\ Functional Nanomaterials, Suranaree University of Technology, Nakhon Ratchasima 30000, Thailand \\ ${ }^{4}$ Division of Superconductivity and Magnetism, University of Leipzig, D-04103 Leipzig, Germany \\ ${ }^{5}$ Laboratory for Neutron Scattering, Paul Scherrer Institut, CH-5232 Villigen, Switzerland
}

(Received 25 February 2014; accepted 6 June 2014; published online 17 July 2014)

\begin{abstract}
Multiferroics in spintronics have opened up opportunities for future technological developments, particularly in the field of ferroelectric (FE)-ferromagnetic (FM) oxide interfaces with functionalities. We find strong exchange bias shifts (up to $84 \mathrm{Oe}$ ) upon field cooling in metal-oxide $\left(\mathrm{Fe} / \mathrm{BaTiO}_{3}\right)$ films combining FM and FE layers. The saturation magnetic moment of the FM layer is also significantly higher than in bulk $\left(3.0 \pm 0.2 \mu_{\mathrm{B}} /\right.$ atom) and the reversal mechanism occurs via a domain nucleation process. X-ray absorption spectroscopy at the Fe K-edge and Ba L3-edge indicate presence of few monolayers of antiferromagnetic $\mathrm{FeO}$ at the interface without the formation of any $\mathrm{BaFeO}_{3}$ layer. Polarized neutron reflectometry corroborates with our magnetization data as we perform depth profiling of the magnetic and structural densities in these bilayers. Our first principles density functional calculations support the formation of antiferromagnetic $\mathrm{FeO}$ layers at the interface along with an enhancement of Fe magnetic moments in the inner ferromagnetic layers. (C) 2014 AIP Publishing LLC. [http://dx.doi.org/10.1063/1.4885316]
\end{abstract}

Recently, it became possible to fabricate composites of ferroelectrics (FE) and ferromagnets (FM) in the form of artificial heterostructures. ${ }^{1-4}$

Exchange bias effect ${ }^{5}$ is a result of exchange anisotropy between two materials with magnetic interaction that are competing with each other. Even though exchange coupling is generally owed to the coupling between a FM and antiferromagnet $(\mathrm{AF})$, unusual and exotic exchange biasing are not uncommon. ${ }^{6,7}$ In contrast to bulk materials, artificial multiferroic heterostructures can be engineered such that a large magnetoelectric response can be obtained even above room temperature.

Here, we report on the unexpected exchange coupling between FE oxide and FM and its consequence on the magnetic structure profile in a $\mathrm{FE}$ oxide $\left(\mathrm{BaTiO}_{3}(\mathrm{BTO})\right)$-metallic $\mathrm{FM}(\mathrm{Fe})$ system. Perovskite oxides such as BTO are ideal candidates for the creation of competitive interactions leading to many ground states at the heterostructural interface. Here, the interface magnetism can be responsible for such exotic phenomena. Magnetism (antiferromagnetism or magnetism with larger anisotropy) at the interface could be induced due to defects in BTO or orbital reconstruction at the interface. The microscopic picture of the magnetism in buried interfaces is the key issue.

We have applied element specific X-ray absorption spectroscopy (XAS) at various elemental edges in exploring the issue. We have also combined this with the depth dependent magnetic and structural density profiles of the metal-oxide films using depth sensitive polarized neutron reflectivity technique (PNR). We propose various theoretical

\footnotetext{
${ }^{\text {a) }}$ Author to whom correspondence should be addressed. Electronic mail: amitesh.paul@frm2.tum.de
}

scenarios within the density functional theory in explaining the observed coupling at low temperatures.

The FE-FM samples in this investigation were prepared by an rf-dc magnetron sputtering chamber on $\mathrm{SrTiO}_{3}$ (STO) [100] substrates. The details of the sample preparation and measurement techniques can be found in the supplemental materials. ${ }^{9}$

X-ray reflectivity (XRR) patterns are measured using the D5000 of Fe-BTO bilayer samples with increasing thickness of the BTO layer grown on STO [100] are shown in Figure 1(a). The shifted Kiessig fringes reflect the varying BTO thickness. The samples are labelled as BTO-4 (15 s), BTO-8 (30 s), BTO-12 (60 s), BTO-20 (100 s), and BTO$1500(12000 \mathrm{~s})$. The numbers in brackets denote the deposition times (and the sample numbers roughly denote the BTO unit cells $\perp$ to the sample-plane) which are roughly proportional to the thickness $\mathrm{t} \pm 5 \AA$ of the BTO layer $(\mathrm{t}=17$ : BTO- $4, \mathrm{t}=32$ : BTO- $8, \mathrm{t}=46$ : BTO- $12, \mathrm{t}=80$ : BTO-20, $\mathrm{t}=6000$ : BTO-1500). The Fe layer on top has a thickness of $\approx 45 \pm 5 \AA$. Atomic and Magnetic force microscopy (AFM/ MFM) was carried out on some representative samples to investigate the morphology and magnetic domains. The AFM pictures (not shown) do not support columnar structure for BTO, thus negating the possibility of a mixed $\mathrm{TiO}_{2} / \mathrm{SrO}$ termination at the FM-FE interface.

Figure 1(b) shows the room-temperature diffraction (XRD) patterns from the $\mathrm{Fe} / \mathrm{BTO}$ samples and the STO substrate. An in-plane $\phi$ scan from D500 is shown in Figure 1(c). We did a systematic variation of the inclination angle $\chi$ to map the intensity variation from the [001] plane to [111] plane of BTO. The fourfold symmetry of the STO[111] reflections along with the fourfold symmetry of the BTO[111] in-plane reflection without any relative shift proves the epitaxial growth 

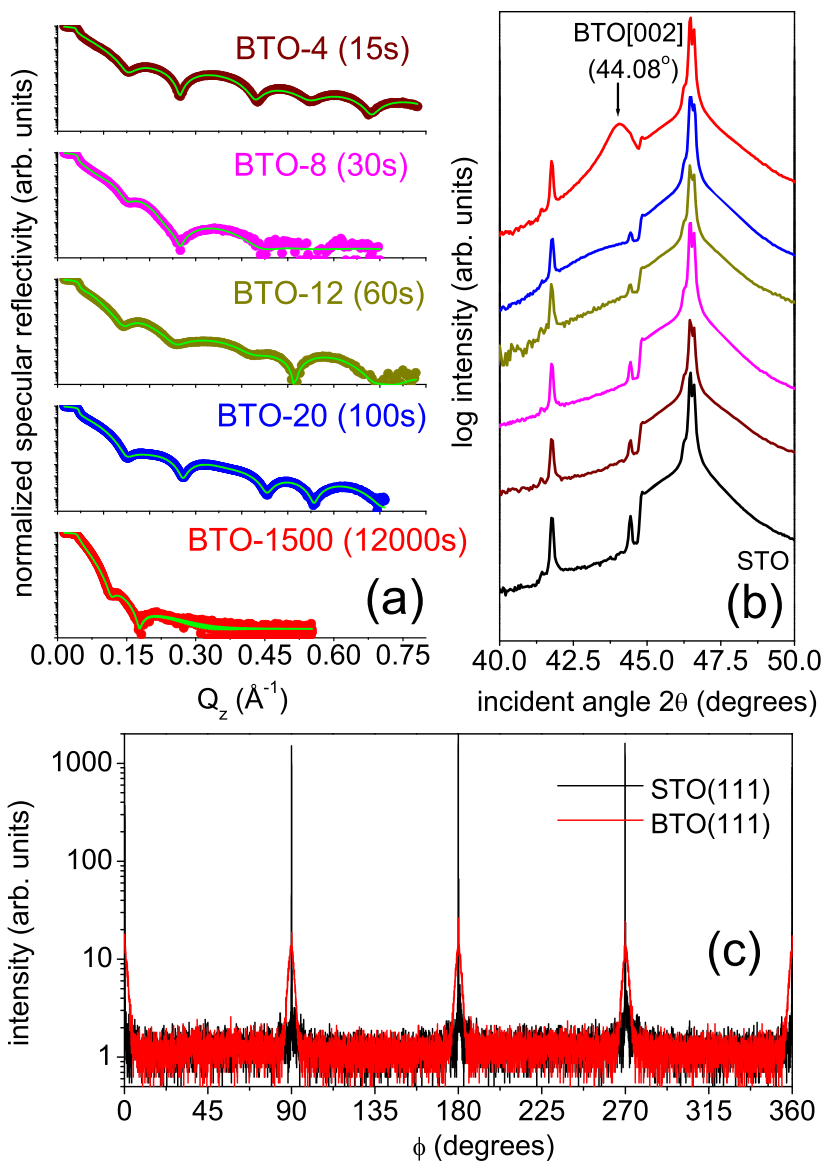

FIG. 1. (a) XRR $\left(\mathrm{Cu}-\mathrm{K}_{\alpha}\right)$ patterns and their fits of [BTO-Fe] bilayers are plotted versus $\vec{Q}_{z}$ at room temperature. (b) XRD patterns of [BTO-Fe] bilayers and the STO substrate. (c) In-plane $\phi$ scans for the STO substrate and the BTO-1500 sample showing the epitaxy of our sputtered samples.

of BTO (at $\Delta \chi=55.17^{\circ}$ inclination to the BTO[001] plane). The XRD pattern from the sample with the thickest BTO layer shows the reflection as typically broad and weak indicating possible $90^{\circ}$ BTO domains. ${ }^{8}$ The out-of-plane lattice parameter $\mathrm{c}=4.105 \AA$ was estimated from the BTO[002] reflection at $44.08^{\circ}$. The in-plane lattice parameter $\mathrm{a}=4.039 \AA$ (at $\chi=34.83^{\circ}$ ) was estimated from the $\mathrm{BTO}[111]$ reflection at $38.37^{\circ}$. This shows that films grow with a relaxed tetragonal structure. Rocking curves around [002] peak for the BTO-1500 sample show a crystallite size of $136 \AA$.

In Figs. 2(a)-2(c), we show the magnetization curve of BTO-8, BTO-12 and BTO-1500 measured at $5 \mathrm{~K}$ with inplane magnetic field after field cooling the samples from above room temperature at +100 Oe. The heterostructures show a distinct enhancement of the coercive fields with the decrease of the temperature and, most significantly, up to $\approx 84$ Oe (BTO-1500) of exchange bias shifts. ${ }^{9}$ One should note that these negative loop shifts $\left(H_{\mathrm{eb}}\right)$ occur irrespective of the thickness of the underlying BTO layer and irrespective of the field cooling directions (we could observe such a loop shift even when we apply a cooling field of $70 \mathrm{kOe}$ ). Such a shift is usually associated with ferromagnetic coupling between the uncompensated moments of an antiferromagnet with the moments of a ferromagnet. More generally, exchange bias shift may also occur due to the development of a domain wall at the interface that does not respond to an external field. We also observe an increase in the $H_{\mathrm{eb}}$ values

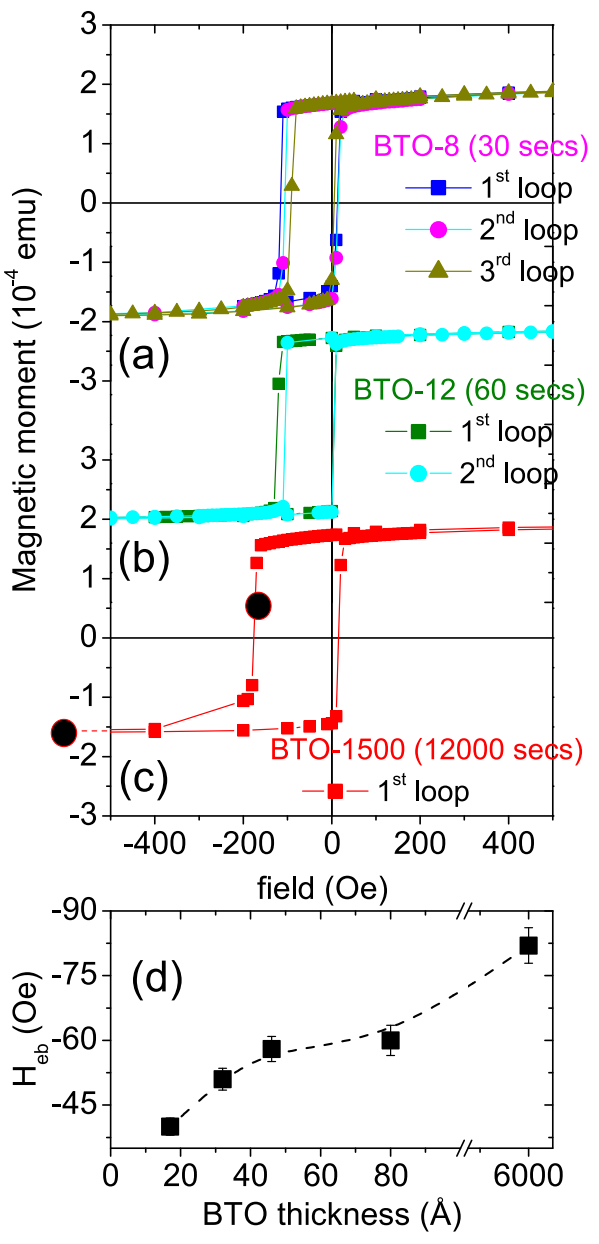

FIG. 2. Hysteresis loop measurements at $5 \mathrm{~K}$ for the (a) BTO-8 bilayer showing the 1 st, 2 nd, and $3 r d$ loop cycles, (b) BTO-12 bilayer showing the 1 st, 2nd loop cycles, and (c) BTO-1500 bilayer showing the $1 s t$, loop cycle. The closed circles in (b) show the fields where the neutron measurements have been performed. (d) Plot of the $H_{\mathrm{eb}}$ versus the thickness of the BTO layer.

with increasing BTO thickness (Fig. 2(d)) which can arise due to (a) variations in the AF layer thickness at the interface or (b) structural changes at the BTO/Fe interface. We also show the $H_{\mathrm{eb}}$ to be persistent even after the 1 st, $2 n d$, and $3 r d$ loop cycles with lowering of the bias field for the BTO-8 sample. This is a typical training effect that has been seen in exchange bias phenomena associated with rearrangements of the uncompensated spins in an AF. ${ }^{10}$ The changes in the coercive field and exchange bias shift show an increase with lowering of the temperature below $100 \mathrm{~K}$. All these observations indicate strongly the existence of an AF layer at the FE-FM interface. It should be noted that we did not find any exchange bias shift in the case of BTO [100]-LSMO samples (grown by pulsed laser deposition) in contrast to the earlier reports on a system where BTO was replaced by BFO (BFO-LSMO). ${ }^{11}$ This indicates that the plausible AF/FM layer at the interface incorporates Fe in BFO-LSMO which is not possible in BTO-LSMO. However, a similar exchange bias was seen for an Fe layer on a STO [100] substrate (deposited simultaneously with BTO-1500) which again points towards an AF layer formation at the interface.

Fig. 3(a) shows a comparison of the measured $\mathrm{Fe}$ K-edge X-ray absorption near-edge structure (XANES) spectra from the samples (solid symbols) and the reference spectra from each of the possible constituents that can produce 
the absorption edge, for example, $\mathrm{Fe}, \mathrm{FeO}, \mathrm{Fe}_{3} \mathrm{O}_{4}$, and $\mathrm{Fe}_{2} \mathrm{O}_{3}$. By considering $\mathrm{Fe}, \mathrm{FeO}, \mathrm{Fe}_{3} \mathrm{O}_{4}$, and $\mathrm{Fe}_{2} \mathrm{O}_{3}$ as the parent components, the XANES spectra of the samples are fitted (lines) with a superposition of XANES profiles of the parent components using the linear combination analysis (LCA) method. The fitting was performed using the package ATHENA $^{12}$ with the LCA tool. The fits are shown in the figure together with the measured XANES spectra. In this way, we estimate the weighted proportions of the constituents in the samples. They are composed of phase-separated regions that differing negligibly in the proportion of their respective constituents $\left(\mathrm{Fe}, \mathrm{FeO}, \mathrm{Fe}_{3} \mathrm{O}_{4}\right.$, and $\mathrm{Fe}_{2} \mathrm{O}_{3}$ ) from sample to sample. The results indicate that there can be small traces of $\mathrm{FeO}(1 \%-2 \%)$ in the samples. The other constituents are $\mathrm{Fe}$ (60\%) with proportions of $\mathrm{Fe}_{2} \mathrm{O}_{3}(18 \%)$ and $\mathrm{Fe}_{3} \mathrm{O}_{4}(20 \%)$.

Fig. 3(b) shows the measured data at the Ba L3-edge for BTO-1500 sample and $\mathrm{BaTiO}_{3}$ single crystal. The ab initio ${ }^{13}$ calculated XANES spectra of $\mathrm{BTO}, \mathrm{BaFeO}_{3}, \mathrm{BaFeO}_{4}$, and $\mathrm{BaFe}_{2} \mathrm{O}_{4}$ are also plotted. For tetragonal BTO, $\mathrm{a}=3.993 \AA$ and $\mathrm{c}=4.033 \AA$ are used as the lattice parameters. Whereas $\mathrm{a}=5.68 \AA$ and $\mathrm{c}=13.86 \AA$ are used for the lattice constant of $\mathrm{BaFeO}_{3}$ (BaFO, hexagonal). For BTO (BaFO), the selfconsistent field muffin-tin atomic potentials calculations are per formed in the spherical cluster radius 5.7(5.2) $\AA$ including 65(50) atoms within the Hedin-Lundqvist exchange potential and the full multiple scattering calculations to include all possible paths within a larger cluster radius of $7 \AA$ (including 120 atoms). Comparing the feature of the spectra, one can easily see that the calculated XANES spectra for BTO are in good

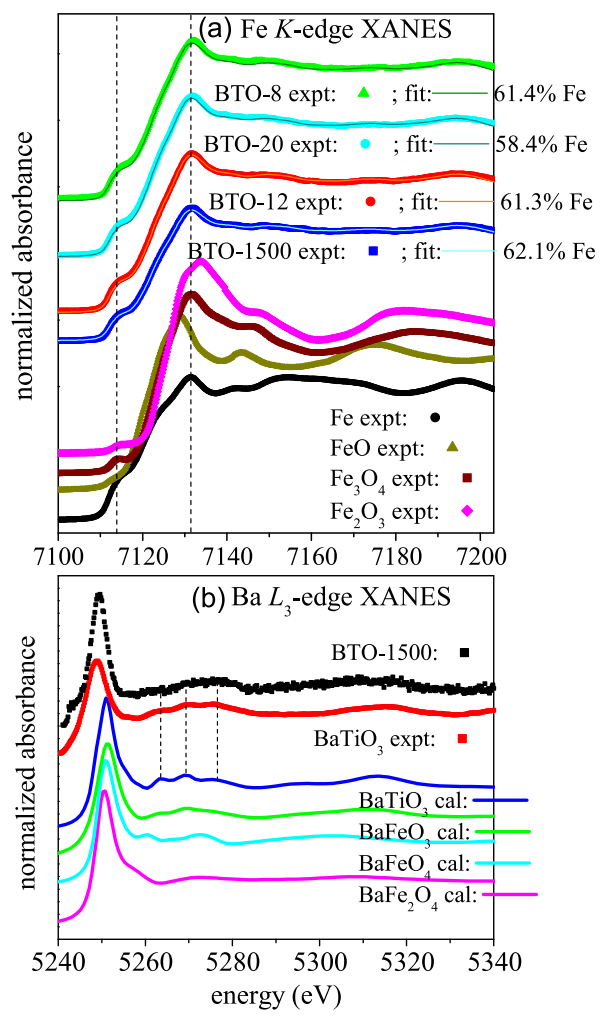

FIG. 3. (a) Comparison of the measured Fe K-edge normalised XANES spectra of the samples (solid symbols) and their fits (lines). Also included are the reference spectra for $\mathrm{Fe}, \mathrm{FeO}, \mathrm{Fe}_{2} \mathrm{O}_{3}$, and $\mathrm{Fe}_{3} \mathrm{O}_{4}$. (b) Comparison of the measured Ba L3-edge spectra of BTO-1500 sample and BTO single crystal. The ab initio calculated XANES spectra of $\mathrm{BTO}$ and $\mathrm{BaFO}_{x}$ are also plotted. agreement with the corresponding features in the measured XANES spectra (both measured and calculated), These results clearly indicate that the formation of $\mathrm{BaFeO}_{x}$ is unlikely.

To this end we further investigated our samples by polarized neutron reflectivity (PNR) which can be exploited to infer on a buried interfacial AF layer and to distinguish it from the top oxide layer. Figures 4(a) and 4(b) display the specular PNR applied from the representative sample BTO-1500 at two different fields $H_{\mathrm{a}}=-150 \mathrm{Oe}$ (close to the coercive field) and $H_{\mathrm{a}}=-1000$ Oe (at saturation field). We chose to show the PNR data from this sample since the possibility of an interfacial AF layer should appear most clear. The sample was field cooled at $1.0 \mathrm{kOe}$ from RT down to $10 \mathrm{~K}$. The average magnetization density in the bilayer is evident from the splitting of the spin-up and spin-down polarized curves $(\mathrm{R}++$ and $\mathrm{R}--)$. The best fits (open symbols) with a simple model of blockpotentials yield average scattering length density (SLD) values. The data from the XRR fits were used to fix the nuclear SLD profile of PNR at RT and then the magnetization profile was optimized. The nuclear $\left(\rho_{\mathrm{n}}\right)$ and magnetic $\left(\rho_{\mathrm{m}}\right)$ components of the SLDs, as obtained from the fitting, are shown in the insets of Figures 4(a) and 4(b). We find a very similar values from all other samples investigated here.

Two most interesting features can be deduced from the fits. (i) The saturation moment of the Fe layer is very high than that in bulk and corresponds to $3.0 \pm 0.2 \mu_{\mathrm{B}} /$ atom. It is independent of temperature $(300-5 \mathrm{~K})$. This is of course reduced to $1.0 \pm 0.2 \mu_{\mathrm{B}} /$ atom when measured close to the coercive field. The magnetization remains always collinear to the applied field $\left(\phi_{\mathrm{A}}=0\right)$. This confirms that the magnetization reversal is via domain nucleation and domain wall

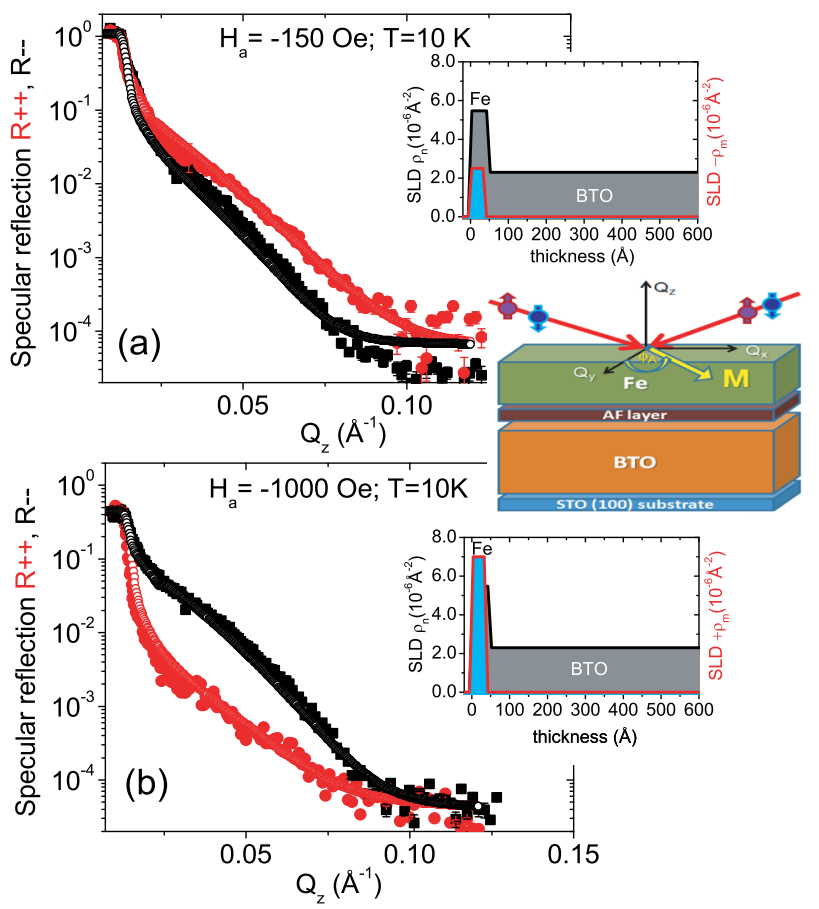

FIG. 4. PNR curves (closed symbols) for BTO-1500 at $10 \mathrm{~K}$ for spin-up and spin-down polarization measured at (a) $H_{\mathrm{a}}=-150 \mathrm{Oe}$ and (b) $H_{\mathrm{a}}=-1000 \mathrm{Oe}$ (saturation) along the decreasing branch of the hysteresis loop after field cooling the sample. The data are plotted versus $\vec{Q}_{z}$ along with the simulations (open symbols). Insets show the depth profiles of the $\rho_{\mathrm{n}}$ (black line) and $\rho_{\mathrm{m}}$ (red line), respectively. A schematic of the PNR measurements is also shown in between the two panels. 
motion. This was also evident from the sharpness of reversal in the hysteresis loop. Note that similar magnetic moment enhancement was found for other samples with lower BTO thickness as well. (ii) In our model, we have considered an interfacial $\mathrm{AF}$ layer of $15 \AA \pm 5$ ( $\sim 10$ monolayers) in order to obtain good agreement between the fits and the data. Note that this information using PNR is unique in the sense that here we can access the buried interfacial layer unlike other surface sensitive techniques involving x-rays or magneto-optic methods. The spin asymmetry (SA) is defined as the ratio of the difference and sum of the non spin flip (NSF) reflectivities, which is most sensitive to the magnetization profile. In order to justify our consideration of few monolayers of (a) an AF interfacial layer we shown in Figure 5 the SA profile where we compare the situation with (b) no AF layer, (c) a Fe layer with reduced $\left(0.4 \mu_{\mathrm{B}} /\right.$ atom) magnetization and (d) an $\mathrm{AF}$ oxide layer at the top instead of at the interface. These simulations clearly indicate that no other possible scenario can match the data more other than that considering an AF layer at the interface. A surface oxidation of composite multiferroics is possible, but it will not significantly alter the magnetic properties at the interface. ${ }^{14}$ One may note that the maximum $H_{\mathrm{eb}}$ values for the $\mathrm{Fe} /$ BTO interface is around $84 \mathrm{Oe}$. The Néel temperature for $\mathrm{FeO}$ is around $200 \mathrm{~K}$. The presence of residual oxygen following the growth of BTO can result in the formation of an antiferromagnetic $\mathrm{FeO}$ layer at the interface. Such an effect has been reported at a $\mathrm{Fe} / \mathrm{MgO}$ interface but with some differences as it depends much on the details of the interfaces. ${ }^{6}$ Uniaxial anisotropy energy increases due to lattice mismatch can raise the blocking temperature. However, we may rule out such possibility as $\mathrm{T}_{\mathrm{B}} \sim 110 \mathrm{~K}$ in the present case. Most significantly, the net magnetization is unexpectedly higher (see PNR) than expected for an Fe layer. The magnetizations changes sharply at the reversal points which indicate a collinear arrangement of moments with respect to the field cooling axis. Hayashi et al. ${ }^{15}$ reported of perovskite $\mathrm{BaFO}$ with a $\mathrm{T}_{c}$ around $110 \mathrm{~K}$ and a saturation magnetic moment of $3.5 \mu_{\mathrm{B}} / \mathrm{Fe}$ atom. However, even if such a secondary layer exits, it is more likely to be a soft ferromagnetic, ${ }^{16}$ at least for a cooling field of $70 \mathrm{kOe}$ and one should not expect an exchange bias loop-shift, as has been observed in the present case.

There are no previous reports of such $\mathrm{FeO}$ layer formation at the Fe/BTO interface. We do not expect to see the $\mathrm{FeO}$

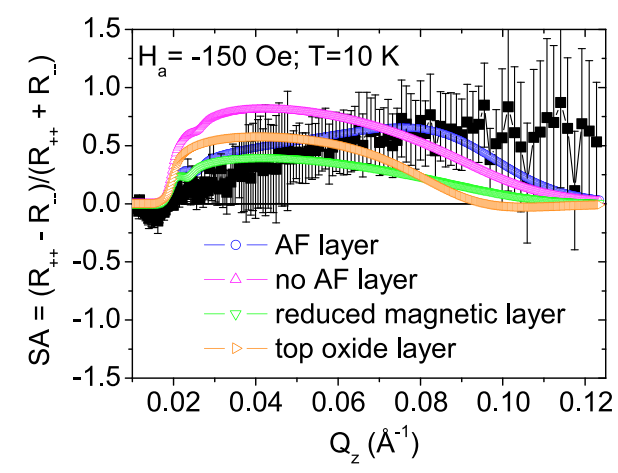

FIG. 5. SA (black symbols) versus $\vec{Q}_{z}$ for BTO-1500 at $10 \mathrm{~K}$ and measured at $H_{\mathrm{a}}=-150 \mathrm{Oe}$ along with the simulations considering (a) an $\mathrm{AF}$ layer (blue circles): [BTO/AF/FM], (b) no AF layer (magenta circles): [BTO/FM], (c) reduced magnetic (FM1) layer (green circles): $[\mathrm{BTO} / \mathrm{FM} 1 / \mathrm{FM}]$ at the interface, and (d) oxide AF layer at the top: [BTO/FM/AF]. at the interface by structural analysis as the lattice parameter of $\mathrm{FeO}$ is $\sim 4.33 \AA$ (close to that of BTO). We calculate the interfacial exchange energy $J_{\text {int }}=0.062 \mathrm{ergs} / \mathrm{cm}^{2}$ which is given by $H_{\mathrm{eb}} M_{\mathrm{FM}} t_{\mathrm{FM}}$, where $M_{\mathrm{FM}}$ is the saturation magnetization and $t_{\mathrm{FM}}$ is the thickness of the FM layer. ${ }^{10} \mathrm{We}$ further calculate the interlayer exchange coupling $\mathrm{J}\left[=J_{\text {int }}(4.33)^{2} / \mathrm{S}_{1} \mathrm{~S}_{2}\right]$ as $5.81 \times 10^{-17}$ ergs with $S_{1}=1$ and $S_{2}=2$ as the atomic spin vectors of $\mathrm{Fe}$ and $\mathrm{FeO}$, respectively. This is close to the value reported for fully compensated $\mathrm{Fe} / \mathrm{FeO}$ interface. ${ }^{17}$

To understand the origin of the observed magnetic properties, we have performed first-principles density functional calculations of the interfacial magnetism by using the plane wave $\mathrm{VASP}^{18}$ package for $\mathrm{Fe} / \mathrm{BTO}$ and $\mathrm{Fe} / \mathrm{FeO} / \mathrm{BTO}$ heterostructures. We used a plane wave energy cut-off of $500 \mathrm{eV}$ and a $k$-point grid of $8 \times 8 \times 1$ for the calculation of the total energies and electronic structures. The Perdew-BurkeErnzerhof parametrization of the generalized gradient approximation ${ }^{19}$ for the exchange correlation was used along with on-site Coulomb interaction $U$ for the $3 d$ orbitals. ${ }^{20} \mathrm{We}$ considered the values of $U=2 \mathrm{eV}$ for $\mathrm{Fe}$ atoms and $5 \mathrm{eV}$ for the $\mathrm{Ti}$ atoms. ${ }^{21} U=4.3 \mathrm{eV}$ for $\mathrm{Fe}$-d orbitals was chosen for the $\mathrm{FeO}$ layers, ${ }^{22}$ as well for $\mathrm{Fe}$ at the interface layer of $\mathrm{Fe} / \mathrm{BTO}$ to compare the results. The supercells were built up with 5 layers of BTO and 9 monolayers of Fe along the $c$-axis and a $\sqrt{2} a \times \sqrt{2} a$ in the $a b$-plane with two $2 \mathrm{Ti}$ atoms as shown in Fig. 6(a). We constructed the supercell so that at each interface, $\mathrm{O}$ atoms of the $\mathrm{TiO}_{2}$ termination occupy atop sites of Fe. Such a termination was shown to be the most stable one. ${ }^{23}$ Recently, a more complicated interface was proposed with the formation of an interface layer with

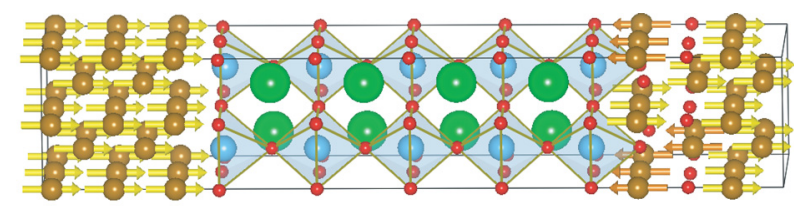

(a)

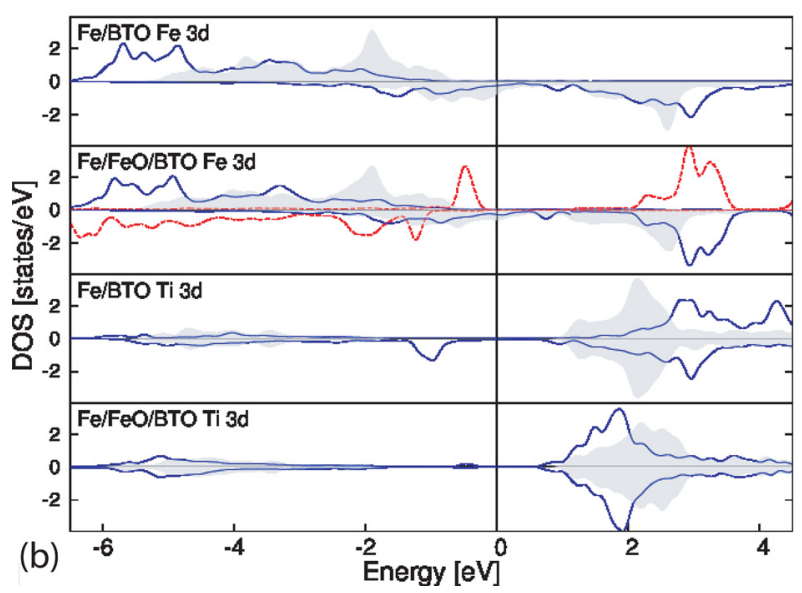

FIG. 6. (a) Ground state magnetic structure for the $\mathrm{Fe} / \mathrm{FeO} / \mathrm{BTO}$ system with two $\mathrm{FeO}$ layers. The yellow arrows indicate the spins up on $\mathrm{Fe}$ and $\mathrm{Ti}$ atoms, while the orange arrows indicate the spin down. $\mathrm{Ba}, \mathrm{Ti}, \mathrm{O}$, and $\mathrm{Fe}$ atoms are shown as green, blue, red, and brown balls, respectively. (b) Density of states resolved for $\mathrm{Ti} 3 \mathrm{~d}$ and $\mathrm{Fe} 3 \mathrm{~d}$ in $\mathrm{Fe} / \mathrm{BTO}$ and $\mathrm{Fe} / \mathrm{FeO} / \mathrm{BTO}$ heterostructures. In each panel, majority and minority spin channels are plotted in the upper and lower half, respectively. Solid lines refer to the interface atoms while the dashed lines refer to the AF coupled $\mathrm{Fe}$ atoms in $\mathrm{Fe} / \mathrm{FeO} /$ BTO. The shaded plots correspond to the atoms in the inner layers. The Fermi level is set to zero. 
stoichiometry $\mathrm{Fe}_{3} \mathrm{O},{ }^{24}$ however this interface is not compatible with the presence of $\mathrm{FeO}$ in the correct stoichiometry. In all our calculations, structural relaxations of the internal degrees of freedom were performed until the HellmannFeynman forces on each atom were less than $10 \mathrm{meV}^{-1}$.

It is already known that the Fe/BTO heterostructure has a ferromagnetic order. ${ }^{21,23}$ When one layer of oxygen is added at the interface to create one $\mathrm{FeO}$ layer, the ground state is still ferromagnetic. However, the oxygen atoms at the interface receive two electrons from the iron interface to realize $\mathrm{Fe}^{+2}$, and hence, the magnetic moment is increased similar to bulk $\mathrm{FeO}$. The magnetic moment in the $\mathrm{FeO}$ layer increases from 3.2 to $3.7 \mu_{\mathrm{B}}$, and the magnetic moment on $\mathrm{Ti}$ is reduced to $0.1 \mu_{\mathrm{B}}$. The average magnetic moment of the iron atoms in the inner layers increases from 2.73 to $2.82 \mu_{\mathrm{B}}$ due to the large moment of the $\mathrm{FeO}$ layers that induce a larger magnetic moment in the $\mathrm{Fe}$ inner layers. When two layers of oxygen atoms are added at the interface, the ground state is composed by one AF interface layer while the other $\mathrm{FeO}$ layer is ferromagnetic. The energy difference $E_{A F}-E_{F M}$ is $209 \mathrm{meV}$ per interface $\mathrm{Fe}$ atom for the $\mathrm{Fe} / \mathrm{BTO}$ heterostructures, while it becomes $-51 \mathrm{meV}$ after the addition of $2 \mathrm{FeO}$ layers. For the latter case, the magnetic order is composed by ferromagnetic chains, antiferromagnetically coupled to each other as shown in Figure 6(a). Another AF phase was found at the surface of these heterostructures. ${ }^{25}$ However, the electrons in the AF surface are localized by the reduction of the dimensionality and hence by the reduction of the bandwidth. In the present case, the localization of the $\mathrm{Fe}-3 \mathrm{~d}$ electrons is due to the $\mathrm{Fe}^{+2}$ valence.

The exchange bias generates an effective magnetic field that increases the magnetization. This effect is observed at the interface between $\mathrm{Fe}$ and $\mathrm{FeO}$ in the $\mathrm{Fe} / \mathrm{FeO} / \mathrm{BTO}$ heterostructure, while is not observed in $\mathrm{Fe} / \mathrm{FeO}$ bilayers that show superparamagnetic behavior at the interfaces. ${ }^{17}$ We propose that the $\mathrm{FeO} / \mathrm{BTO}$ interface enhances the magnetocrystalline anisotropy, dominating over the thermal energy responsible for the superparamagnetic phase as already observed in $\mathrm{Co} / \mathrm{CoO}$ bilayers. ${ }^{26}$ As shown in Figure 6(b), the Fe interface is halfmetallic in $\mathrm{Fe} / \mathrm{BTO}$ while in $\mathrm{Fe} / \mathrm{FeO} / \mathrm{BTO}$, the AF coupled $\mathrm{Fe}$ atoms show an insulating character as in bulk $\mathrm{FeO}^{22}$ The Ti $3 \mathrm{~d}$ orbitals are mainly placed at $2 \mathrm{eV}$ above the Fermi energy with a peak below the Fermi level in the spin-down channel that produces a finite magnetic moment in $\mathrm{Fe} / \mathrm{BTO}$, while the magnetic moment on $\mathrm{Ti}$ is suppressed in $\mathrm{Fe} / \mathrm{FeO} / \mathrm{BTO}$.

In conclusion, we have observed and analyzed enhanced magnetic moment and exchange bias effects in ferroelectricferromagnetic $\mathrm{Fe} / \mathrm{BaTiO}_{3}$ bilayers. This reflects the presence of uncompensated spins in the Fe-BTO interface. Polarized neutron reflectometry measurements reveal that this effect originates from the presence of antiferromagnetic $\mathrm{FeO}$ layers at the interface between ferromagnetic $\mathrm{Fe}$ and $\mathrm{BaTiO}_{3}$. X-ray absorption spectroscopy agrees well with the $\mathrm{FeO}$ formation and do not indicate a BaFO layer. Our first principles density functional calculations support the formation of antiferromagnetic $\mathrm{FeO}$ layers at the interface, while the absence of $\mathrm{FeO}$ renders the system ferromagnetic. The enhanced magnetic moment of $\mathrm{Fe}$ in experiments is also observed in theory due to moment induced by $\mathrm{FeO}$ at the interface. One can envisage that as ferroelectric domain structure in BTO is manipulated by an electric field, electrical manipulation of exchange bias would also be possible in such systems.

We would like to thank J. Mannhart, A. Herrnberger, T. Mairoser, and A. Schmehl for the collaboration during the course of realizing the sample deposition chamber at the University of Augsburg for the Transregional Collaborative Research Center TRR 80 of the Deutsche Forschungsgemeinschaft. C.A. and B.S. acknowledge financial support from Carl Tryggers Stiftelse (Grant No. CTS 12:419). Also supercomputing time allocation from Swedish National Infrastructure for Computing is gratefully acknowledged.

${ }^{1}$ H. Zheng, J. Wang, S. E. Lofland, Z. Ma, L. Mohaddes-Ardabili, T. Zhao, L. Salamanca-Riba, S. R. Shinde, S. B. Ogale, F. Bai, D. Viehland, Y. Jia, D. G. Schlom, M. Wuttig, A. Roytburd, and R. Ramesh, Science 303, 661 (2004).

${ }^{2}$ N. Reyren, S. Thiel, A. D. Caviglia, L. Fitting Kourkoutis, G. Hammerl, C. Richter, C. W. Schneider, T. Kopp, A.-S. Rüetschi, D. Jaccard, M. Gabay, D. A. Muller, J.-M. Triscone, and J. Mannhart, Science 317, 1196 (2007).

${ }^{3}$ J. Chakhalian, J. W. Freeland, H. U. Habermeier, G. Cristiani, G. Khaliullin, M. Veenendaalvan, and B. Keimer, Science 318, 1114 (2007).

${ }^{4}$ S. M. Wu, S. A. Cybart, P. Yu, M. D. Rossell, J. X. Zhang, R. Ramesh, and R. C. Dynes, Nature Mater. 9, 756 (2010).

${ }^{5}$ W. H. Meiklejohn and C. P. Bean, Phys. Rev. 102, 1413 (1956).

${ }^{6}$ Y. Fan, K. J. Smith, G. Lupke, A. T. Hanbicki, R. Goswami, C. H. Li, H. B. Zhao, and B. T. Jonker, Nat. Nanotechnol. 8, 438 (2013).

${ }^{7}$ M. Gibert, P. Zubko, R. Scherwitzl, J. Íñiguez, and J.-M. Triscone, Nature Mater. 11, 195 (2012).

${ }^{8}$ X. Moya, L. E. Hueso, F. Maccherozzi, A. I. Tovstolytkin, D. I. Podyalovskii, C. Ducati, L. C. Phillips, M. Ghidini, O. Hovorka, A. Berger, M. E. Vickers, S. S. Dhesi, and N D Mathur, Nature Materials 12, 52 (2013).

${ }^{9}$ See supplementary material at http://dx.doi.org/10.1063/1.4885316 for the stability and the effect of phase transition of the BTO layer with temperature for various thicknesses and the temperature dependence of the magnetization.

${ }^{10}$ A. Paul, C. Schmidt, N. Paul, A. Ehresmann, S. Mattauch, and P. Böni, Phys. Rev. B 86, 094420 (2012).

${ }^{11}$ M. Huijben, P. Yu, L. W. Martin, H. J. A. Molegraaf, Y.-H. Chu, M. B. Holcomb, N. Balke, G. Rijnders, and R. Ramesh, Adv. Mater. 25, 4739-4745 (2013).

${ }^{12}$ B. Ravel and M. Newville, J. Synchrotron Radiat. 12, 537 (2005).

${ }^{13}$ A. L. Ankudinov, B. Ravel, J. J. Rehr, and S. D. Conradson, Phys. Rev. B 58, 7565 (1998).

${ }^{14}$ M. Fechner, S. Ostanin, and I. Mertig, Phys. Rev. B 80, 094405 (2009).

${ }^{15} \mathrm{~N}$. Hayashi, T. Yamamoto, H. Kageyama, M. Nishi, Y. Watanabe, T. Kawakami, Y. Matsushita, A. Fujimori, and M. Takano, Angew. Chem., Int. Ed. 50, 12547 (2011).

${ }^{16}$ S. Chakraverty, T. Matsuda, N. Ogawa, H. Wadati, E. Ikenaga, M. Kawasaki, Y. Tokura, and H. Y. Hwang, Appl. Phys. Lett. 103, 142416 (2013).

${ }^{17}$ X. Lin, A. S. Murthy, G. C. Hadjipanayis, C. Swann, and S. I. Shah, J. Appl. Phys. 76, 6543 (1994).

${ }^{18}$ G. Kresse and D. Joubert, Phys. Rev. B 59, 1758 (1999).

${ }^{19}$ J. P. Perdew, K. Burke, and M. Ernzerhof, Phys. Rev. Lett. 77, 3865 (1996).

${ }^{20}$ A. I. Liechtenstein, V. I. Anisimov, and J. Zaanen, Phys. Rev. B 52, R5467 (1995).

${ }^{21}$ D. Di Sante, K. Yamauchi, and S. Picozzi, J. Phys.: Condens. Matter 25, 066001 (2013).

${ }^{22}$ M. Cococcioni and S. de Gironcoli, Phys. Rev. B 71, 035105 (2005).

${ }^{23}$ C.-G. Duan, S. S. Jaswal, and E. Y. Tsymbal, Phys. Rev. Lett. 97, 047201 (2006).

${ }^{24}$ L. Bocher, A. Gloter, A. Crassous, V. Garcia, K. March, A. Zobelli, S. Valencia, S. Enouz-Vedrenne, X. Moya, N. D. Marthur, C. Deranlot, S. Fusil, K. Bouzehouane, M. Bibes, A. Barthélémy, C. Colliex, and O. Stéphan, Nano Lett. 12, 376-382 (2012).

${ }^{25}$ M. Fechner, I. V. Maznichenko, S. Ostanin, A. Ernst, J. Henk, P. Bruno, and I. Mertig, Phys. Rev. B 78, 212406 (2008).

${ }^{26}$ V. Skumryev, S. Stoyanov, Y. Zhang, G. Hadjipanayis, D. Givord, and J. Nogués, Nature 423, 850 (2003). 